NOTICE: This is the author's version of a work that was accepted for publication in Chemical Engineering Science. Changes resulting from the publishing process, such as peer review, editing, corrections, structural formatting, and other quality control mechanisms may not be reflected in this document. Changes may have been made to this work since it was submitted for publication. A definitive version was subsequently published in Chemical Engineering Science, Vol. 95. (2013). doi: 10.1016/j.ces.2013.03.036 


\title{
Multi-scale models for the optimization of batch bioreactors
}

\author{
Emily Wan-Teng Liew ${ }^{\text {a }}$ Jobrun Nandong ${ }^{\text {a }}$, Yudi Samyudia ${ }^{\text {a,* }}$ \\ ${ }^{a}$ Department of Chemical Engineering, Curtin University, CDT 250, 98009 Miri, Sarawak, \\ Malaysia
}

Email: emily.liew@curtin.edu.my, jobrun.n@curtin.edu.my,yudi.samyudia@curtin.edu.my

*Corresponding author: Yudi Samyudia

Tel: +6085-443939; Fax: +6085-443837; email: yudi.samyudia@curtin.edu.my

\begin{abstract}
Process models play an important role in the bioreactor design, optimisation and control. In previous work, the bioreactor models have mainly been developed by considering the microbial kinetics and the reactor environmental conditions with the assumption that the ideal mixing occurs inside the reactor. This assumption is relatively difficult to meet in the practical applications. In this paper, we propose a new approach to the bioreactor modelling by expanding the so-called Herbert's Microbial Kinetics (HMK) model so that the developed models are able to incorporate the mixing effects via the inclusion of the aeration rate and stirrer speed into the microbial kinetics. The expanded models of Herbert's microbial kinetics allow us to optimize the bioreactor's performances with respects to the aeration rate and stirrer speed as the decision variables, where this optimisation is not possible using the original HMK model of microbial kinetics. Simulation and experimental studies on a batch ethanolic fermentation demonstrates the use of the expanded HMK models for the optimisation of bioreactor's performances. It is shown that the integration of the expanded HMK model with the Computational Fluid Dynamics (CFD) model of mixing, which we call it as a Kinetics Multi-Scale (KMS) model, is able to predict the experimental values of yield and productivity of the batch fermentation process accurately (with less than 5\% errors).
\end{abstract}

Keywords: Bioreactor; CFD; Fermentation; Kinetics; Modelling; Optimisation 


\section{Introduction}

Bioreactors are widely used in process industries for mixing and blending of liquids for biochemical reactions (Harvey and Rogers, 1996; Rahimi and Parvareh, 2005). Bioreactors used in biotechnological applications are commonly designed to meet the requirements of the microorganism cell-culture environment by addressing the key variables such as temperature, oxygen, $\mathrm{pH}$, nutrients, metabolites and biologically active molecules (Hutmacher and Singh, 2008). The bioreactor's performances are characterized by its transport capacities in order to optimally supply the microorganism with the required nutrients during the fermentation process (Lübbert, 1992).

It is very common that the bioreactors are equipped with impellers such that they operate in the turbulent flow regime in order to improve the mixing conditions. The presence of such mixing conditions often makes the task of bioreactor optimisation difficult (Ranade, 1997). It has been recognized that the awareness of the non-uniformity distribution of the intensity and quality of flow, turbulent kinetic energy, turbulent eddies and concentrations of species involved throughout the bioreactor is essential to devise an efficient operational strategies of bioreactors, not only to achieve good yield but also consistent product quality (Venneker et al., 2002).

From systems engineering perspective, mathematical modelling has been one of the most successful scientific tools available to improve the performance of a bioreactor via the improvement of the metabolic capabilities of microorganism by mean of genetic manipulations of the cell metabolism and the bioprocess conditions (Wiechert, 2002). Hence, process models would become more pervasive in the design, optimisation and control of bioreactors (Jiang et al., 2002).

The common approach in the bioreactor design, optimisation and control has always relied on the kinetics of fermentation, which assumes well-mixing behaviour. Our previous studies, for example Liew et al. (2009) showed that the deviation from the ideal mixing behaviour (i.e. non-ideal mixing phenomena) could lead to severe loss in yield and changes in microbial physiology. The integration of mixing phenomena into the bioreactor modelling is therefore vital, but it is not an easy task because the detailed description of the turbulent flow field, in combination with other transport equations, needs to be addressed for the interactions of mixing and fluid flow (Jenne and Reuss, 1999). 
The mixing is particularly important in the case of such practical applications as the industrial fermentation processes (Bezzo et al., 2003). It is also important to note that such a fermentation process can be highly sensitive to other variables such as batch time, liquid volume and initial nutrient concentrations due to their effects on cellular metabolism. The optimisation of bioreactors now implies the manipulation of both microbial culture and the environmental factors involved, i.e. a multivariable optimisation of the process (Konde and Modak, 2007). Model-based optimisation is therefore a vital tool in determining the batch operating strategies (Hjersted and Henson, 2006). One of the fundamental aspects to the success of the model-based optimisation is the model of microbial kinetics adopted, which must accurately capture the effects of decision variables $(\mathrm{pH}$, temperature, aeration rate and stirrer speed) on the rates of growth, substrate consumption and product formation.

To date, most of the currently available models of microbial kinetics do not capture the effects of the aeration rate (AR) and stirrer speed (SS) on the rates of growth, substrate consumption and product formation. Therefore, the main contribution of this paper lies in the development of bioreactor models, where three approaches to incorporating the effects of AR and SS into the model of microbial kinetics based on the Herbert's concepts are proposed. Two of the three developed models are referred to in this paper as the expanded Herbert's Microbial Kinetics (HMK) models. The proposed approaches can also be extended to any other conventional model of microbial kinetics if we want to incorporate the fluid mixing phenomena.

The objectives of this paper are two folds. The first objective is to propose three modelling approaches to capturing the mixing mechanism so that AR and SS can be used as parameters to optimize the bioreactor's performances, i.e. yield and productivity. The second objective is to determine the optimal values of AR and SS for a batch fermentation process by applying the Response Surface Methodology (RSM) to maximize the fermentation yield and productivity using the developed models. Simulation and experimental studies were performed to validate the results of this work. 


\section{Material and methods}

\subsection{Microorganism and inoculum}

In this study, a set of experiments was conducted using the BIOSTAT A-plus 2L, MOAssembly bioreactor operated at batch mode. Rushton turbine is used as the agitation system, whereas air sparger is utilized as the aeration system. Rushton turbine is a disc turbine, which has been considered as the optimum design for use in many fermentation processes (Stanbury et al., 1995). This kind of disc turbine is most suitable in a bioreactor since it could break up a fast air stream without itself becoming flooded in air bubbles. The industrial Baker's yeast was utilized as the inoculum culture with glucose as the substrate. The inoculum was grown in a $250 \mathrm{~mL}$ conical flask and was incubated at room temperature for 8 hours.

\subsection{Growth medium}

First, $1.5 \mathrm{~L}$ of fermentation medium was prepared by adding $75 \mathrm{~g}$ glucose, $7.5 \mathrm{~g}$ yeast, $3.75 \mathrm{~g}$ $\mathrm{NH}_{4} \mathrm{Cl}, 4.37 \mathrm{~g} \mathrm{Na}_{2} \mathrm{HPO}_{4}, 4.5 \mathrm{~g} \mathrm{KH}_{2} \mathrm{PO}_{4}, 0.38 \mathrm{~g} \mathrm{MgSO}$, $0.12 \mathrm{~g} \mathrm{CaCl}_{2}, 6.45 \mathrm{~g}$ citric acid and 4.5g sodium citrate. The medium culture was sterilized at $121^{\circ} \mathrm{C}$ for 20 minutes and then cooled down to room temperature. Then, $40 \mathrm{~mL}$ of inoculum was added to the fermentation medium. The temperature and $\mathrm{pH}$ were maintained at $30^{\circ} \mathrm{C}$ and $\mathrm{pH} 5$, respectively. The batch process was stopped after 72 hours and the samples were taken at every 2-4 hours of sampling interval and were analyzed for glucose and ethanol concentrations. The experiments were repeated at various values of AR and SS within the range of 1.0-1.5LPM of AR and 150250rpm of SS i.e. $[1.0,150.0]^{T} \leq\left[\begin{array}{ll}A R & S S\end{array}\right]^{T} \leq[1.5,250.0]^{T}$. Therefore, the base-line condition or the mid-point of these ranges of AR and SS is at 1.25LPM of AR and 200rpm of SS.

\subsection{Glucose and ethanol concentrations}

Each sample was first filtered, and then analyzed for the concentrations of substrate and ethanol using R-Biopharm test kits and UV spectrophotometer under a wavelength of $340 \mathrm{~nm}$, as outlined in the procedures provided by the test kits. All samples were tested at room temperature. 


\subsection{Optical Density}

Each sample was first filtered. The optical density was then analyzed by using UV spectrophotometer under a wavelength of $340 \mathrm{~nm}$, similar wavelength utilized for the analysis of glucose and ethanol concentrations, in order to show consistency of concentrations analysis. No test kits were required as the UV spectrophotometer could directly analyze the optical density. All samples were tested under room temperature.

\subsection{Experiment designs for response surface methodology}

In this study, we adopt the Central Composite Design (CCD) for the design experiments since this design provides a solid foundation for the generation of a response surface map. To create a CCD, it is important to locate new points along the axes of the factor space. Table 1 shows the CCD matrix employed for both AR and SS and the corresponding values of yield and productivity. For maximum efficiency, the axial or star points are to be located at a specific distance outside the original factor range. In the application of Response Surface Methodology, the regression analysis is employed to describe the experimental data collected. The least square technique is used to fit the model equation containing the input variables by minimizing the residual errors of the sum of squared deviations between the experiments and the estimated responses.

In our study, a two-factor factorial design is selected since AR and SS are the two factors of interest to study on the effect of bioreactor performance. $\mathrm{AR}\left(X_{1}, \mathrm{LPM}\right)$ and SS $\left(X_{2}, \mathrm{rpm}\right)$ are considered as input variables. Yield $\left(Y_{1}, \%\right)$ and productivity $\left(Y_{2}, \mathrm{~g} / \mathrm{L} . \mathrm{hr}\right)$ are considered as output variables. The levels of the input variables are selected based on the range of reasonable formulations since the interpretation of the results are valid only within experimental limits in the laboratory available. Three levels are coded as $-1,0$ and +1 , which corresponded to the lower, middle and higher values respectively. The experiments are chosen to realize every possible combination between the variables, with the levels coded. Therefore, Table 1 shows the input variables and levels employed. 
The experiments are randomized in order to make the experimental error as small as possible. For CCD, it is an extension of two-level full factorial design. A CCD enables a quadratic model to be fitted by including new levels. Therefore, there are 2 blocks available in the CCD matrix shown in Table 2. Once all experiments in Block 1 has been completed and all data were recorded, it is required to extend the experiments to Block 2. All experimental data from Block 1 and Block 2 will then be used to develop the mathematical model.

As shown in Table 2, the experimental tests involved fourteen trials. Standard Order is the order of treatment combinations based on the level indicated in Table 2. Run Order is the order of experiments to be carried out. For each experimental trial, the new conditions of the AR and SS were utilized. These results were further analyzed by performing the ANOVA on the residuals for detecting outliers (Noordin et al., 2004).

\section{Bioreactor modelling}

\subsection{Objectives and approaches}

The majority kinetics of ethanol fermentation utilize a formal macro-scale approach to describe the microbial growth, whereby they are empirical and based on either Monod's equation or on its numerous modifications which take into account the inhibition of microbial growth by a high concentration of product and/or substrate (Starzak et al., 1994). The models so far only explained the effect of ethanol inhibition via the mechanism of non-competitive inhibition of a simple reversible enzymatic reaction without taking into consideration of the mixing mechanism occurring inside the bioreactor, i.e. the assumption of well-mixing behaviour is applied. Deviation from the ideal mixing behaviour in practice could lead to severe loss in yield and changes in microbial physiology. Thus, the integration of mixing phenomena (and the effects of AR and SS) is necessary to be taken into account, and this is the objective of our modelling work.

Three modelling approaches to incorporating the effects of mixing in a batch ethanolic fermentation process are therefore proposed in this paper: (1) statistical data-based (SDB) model, (2) kinetics hybrid (KH) model and (3) kinetics multi-scale (KMS) model. To validate the developed models, a series of experimental studies were conducted using the BIOSTAT 
A-plus 2L, MO-Assembly bioreactor operated at batch mode with the ranges of AR of 1.01.5LPM and SS of 150-250rpm.

\subsection{Batch bioreactor model with Kinetics Herbert's concept}

In order to predict the yield $Y$ and productivity $\operatorname{Pr}$, a batch bioreactor can be modelled dynamically as follows:

$$
\begin{aligned}
& \dot{Z}=\left[\begin{array}{c}
\dot{X}_{v} \\
\dot{S} \\
\dot{P}
\end{array}\right]=\left[\begin{array}{c}
r_{x} \\
r_{s} \\
r_{p}
\end{array}\right] \\
& \Phi=\left[\begin{array}{c}
Y \\
P_{r}
\end{array}\right]=\left[\begin{array}{c}
100 \%\left(P\left(t_{b}\right)-P_{0}\right) /\left(S_{0}-S\left(t_{b}\right)\right) \\
\left(P\left(t_{b}\right)-P_{0}\right) / t_{b}
\end{array}\right]
\end{aligned}
$$

where $\dot{Z}$ is the vector of state variables consisting of optical density $\left(\dot{X}_{v}\right)$, substrate $(\dot{S})$ and product $(\dot{P})$ concentrations. $\Phi$ is the vector of performance measure consisting of $Y$ and $P_{r}$.

$S_{0}=S(0)$ and $P_{0}=P(0)$ is the initial substrate and ethanol concentrations $(\mathrm{g} / \mathrm{L})$ of the medium, $\left(t_{b}\right)(\mathrm{hrs})$ is the batch time for the fermentation process. Other variables are the concentration profiles of substrate $(S)$, product (ethanol) $(P)$ and viable cell (optical density) $\left(X_{v}\right)$. The microbial kinetics are: (1) rate of growth $\left(r_{x}\right),(2)$ rate of product formation $\left(r_{p}\right)$ and (3) rate of substrate consumption $\left(r_{s}\right)$.

In this work, the Herbert's concept of endogenous metabolism is adopted since it has been used in numerous studies to describe the kinetics of ethanolic fermentation with sufficient accuracy (Starzak et al. 1994). Moreover, the by-product concentration is not included in this study because we focus on the impacts of AR and SS on the concentration profiles of substrate $S$, product (ethanol) $P$ and viable cell (optical density) $X_{v}$.

The Herbert's concept assumes that the observed rate of biomass formation $\left(r_{x}\right)$ comprised of the growth rate $\left(r_{x}\right)_{g}$ and the rate of endogenous metabolism $\left(r_{x}\right)_{\text {end }}$.

$$
r_{x}=\left(r_{x}\right)_{g}+\left(r_{x}\right)_{\text {end }}
$$

where 
$\left(r_{x}\right)_{g}=\left[k_{1} X_{v} S /\left(k_{2}+S\right)\right] \exp \left(-k_{5} P\right)$

$\left(r_{x}\right)_{\text {end }}=-k_{6} X_{v}$

The rates of substrate consumption $\left(r_{s}\right)$ and product formation $\left(r_{p}\right)$ are assumed to be proportional to the biomass growth rate:

$r_{s}=-k_{3}\left(r_{x}\right)_{g}$

$r_{p}=k_{4}\left(r_{x}\right)_{g}$

Note that the kinetics of ethanolic fermentation based on the Herbert's concept consists of six kinetic model parameters i.e. $\mathrm{K}=\left[\begin{array}{llllll}k_{1} & k_{2} & k_{3} & k_{4} & k_{5} & k_{6}\end{array}\right]^{T}$.

\section{3. $\quad$ Statistical Data-Based (SDB) model}

Rather than using the bioreactor models equations from (1-7), the SDB model is developed by applying a regression analysis to a set of experimental data for different AR, SS, yields $(Y)$ and productivity $\left(P_{r}\right)$. By applying this approach, the effect of mixing arising from different values of AR and SS are included in the regression model by treating the AR and SS as inputs (or experimental variables $X$ ) and the yield $(Y)$ and productivity $\left(P_{r}\right)$ as outputs (or response variables $\Phi)$. After applying design experiments to the bioreactor, sets of experiment data for both inputs and outputs are obtained.

Generally in the development of statistical-based model, it is assumed that there exists a relationship $\mathcal{F}: X \rightarrow \Phi$ where $X \in R^{n}$ is an $n$-dimensional vector of inputs, $\Phi \in R^{m}$ is an $m$ dimensional vector of outputs and $\mathcal{F} \in R^{m}$ is an $m$-dimensional vector of functions space. As $\Phi$ is normally an implicit function of $X$, in real practice it is often difficult to obtain the exact relationship between the input and response vectors especially for complex systems. Thus, one way to develop an explicit relationship between them is by using a regression model where the model parameters are obtained by an optimisation.

Let us consider the regression model as a quadratic model:

$\hat{\Phi}=A+B X+X^{T} D X+\varepsilon$ 
where $A, B, D$ are defined as model parameters, whereby $A, B$ and $D$ will be estimated in such a way that the sum of the squared errors between the predicted performance measure $(\hat{\Phi})$ and experimental values $(\Phi)$ of the responses are minimised. $X$ is the vector of experimental inputs (AR and SS). $\varepsilon$ is the predicted error. So, this problem can be mathematically stated as:

$$
P_{1}: \min \left\{\sum_{i=1}^{k}\left[\left(\hat{\Phi}-\Phi_{i}\right)\right]^{T}\left[\left(\hat{\Phi}_{i}-\Phi_{i}\right)\right]\right\}
$$

where $\hat{\Phi}_{i}$ is the predicted values by Eq. (8) and the subscript $i$ indicates the experimental number. Based on the full factorial design, the total number of experimental runs for $n$ inputs is given by $k=2^{n}$; in our case $n=2$ and hence $k=4$. Note that different values of $k$ result in different experimental designs.

To test the optimisation results of $P_{1}$, the Analysis of Variance (ANOVA) is performed where the results must be significant along with the analysis of curvature. Curvature analysis is vital to indicate whether the experimental results could fit well into the proposed model (Wadsworth, 1998). If the curvature is significant, i.e. the curvature lies in the region of the desired optimum response, the optimisation results are acceptable. If the curvature is insignificant, the optimisation results are not acceptable. A method based on the Path of Steepest Ascent (PSA) (Wadsworth, 1998) is adopted, and where the curvature is further analyzed until it is shown to be significant.

Note that some process constraints such as $X_{\min } \leq X \leq X_{\max }$ where $X_{\min }$ and $X_{\max }$ denote the lower and upper limits of inputs, respectively can be included in the optimisation of $P_{1}$. Furthermore, different model structures can also be selected while solving the optimisation such as linear or second order model as given by:

$$
\begin{aligned}
& \hat{\Phi}=A+B X+\varepsilon, \text { or } \\
& \hat{\Phi}=A+B X+X X^{T} C^{T} \varepsilon
\end{aligned}
$$

where $\hat{\Phi}$ is the predicted values and $C$ is an off-diagonal matrix defined as $C:=\left[\begin{array}{cc}0 & c_{1} \\ c_{2} & 0\end{array}\right]$. 


\subsection{Kinetics Hybrid (KH) model}

The basic assumption underlying the development of Kinetics Hybrid model (Starzak et al., 1994 ) is that the kinetic parameters are the function of the inputs $X$ as:

$\mathrm{K}=h(X, \Theta)$

Here $\Theta \in R^{n_{\theta} \times m_{\theta}}$ is a matrix whose elements correspond to the parameters to be determined later. Therefore substituting Eq. (12) into Eq. (3-7), the microbial kinetics of Herbert's can now be expressed as

$R_{x}=g_{m}(Z, X, \mathrm{~K})$

where $R_{x}$ is the vector of microbial kinetics, i.e. $r_{x},\left(r_{x}\right)_{g}$ and $\left(r_{x}\right)_{e n d} . g_{m}$ is the Herbert Microbial Model and $X$ is the process constraint.

The advantage of expanded Kinetics Herbert's model, i.e. Eq. (13) over the original microbial kinetic model, i.e. Eq. (3-7) is that the expanded one can be directly used to optimize the yield $Y$ and the productivity $\operatorname{Pr}$ with respect to the aeration rate (AR) and stirrer speed (SS).

The development of Kinetics Hybrid model involves two main steps:

1. For experimental run $i$, obtain the kinetics parameters $\kappa_{i}$ using the original kinetics Herbert's model based on Eq. (3-7) and batch reactor model based on Eq. (1-2)

2. For the obtained kinetic parameters $\mathrm{K}_{i}, \forall i \in\{1,2, . . k\}$ and sets of aeration rates $\left(\mathrm{AR}_{i}\right)$ and stirrer speeds $\left(\mathrm{SS}_{i}\right)$, find $\Theta$ in Eq. (12) using regression method

The combination of the batch bioreactor model which is denoted by Eq. (1-2), the Herbert's kinetics model, denoted by Eq. (3-7) and the regression model of Eq. (12) constitutes the socalled kinetics hybrid $(\mathrm{KH})$ model of bioreactor. Clearly, in this approach, the effect of mixing is now embedded into the bioreactor model, i.e. Eq. (1).

In more details, the development of kinetics hybrid model follows the systematic procedure as follows:

Step 1: Identification of Herbert's Kinetic Parameters 
The Herbert's kinetic parameters $\left(k_{1}, k_{2}, \ldots k_{6}\right)$ is obtained via optimisation by solving the following quadratic problem.

$$
P_{2}: \min _{\mathrm{K}}\left\{\Sigma_{j=1}^{q}\left[Z_{j}-\hat{Z}_{j}\right]\right\}
$$

Here $\hat{Z}_{j}$ is the predicted value of $Z$ using the bioreactor model of Eq. (1-2) at the $j$-sample time, and $q$ is the number of samples taken during the course of batch experiments.

For the $i$-experimental run, the corresponding solution to problem $P_{2}$ will yield $\kappa_{i}=\kappa_{i}^{*}$ that minimizes the sum of squared errors between the predicted values and experimental values of $Z_{i}$. For a $k+1$ number of experimental runs, we will obtain a set of $\mathrm{K}=\left\{k_{0}^{*}, k_{1}^{*}, k_{2}^{*}, . . k_{k}^{*}\right\}$, which contains the solutions corresponding to all experimental runs including the base-line experimental run i.e $k_{0}^{*}$.

Step 2: Determine the Regression Model of Eq. (11)

This step is to find the regression model of $\kappa$ in term of $X$. Thus, this is equivalent to finding $\Theta=\Theta^{*}$ where the problem can be stated as

$$
P_{2}: \min _{\Theta}\left\{\Sigma_{i=0}^{j}\left[\kappa_{1}^{*}-\hat{\kappa}_{i}\right]^{T}\left[\kappa_{2}^{*}-\hat{\kappa}_{i}\right]\right\}, \forall \kappa_{i} \in \mathrm{K}
$$

where $j$ is the number of experiment runs based on the design of experiment (i.e. $j=4$ if factorial design is adopted). Here $i=0$, indicates the experiment at the base-line conditions and $\hat{\kappa}$ denotes the predicted value of $\kappa$ based on a regression model, e.g. as Eq. (8), (10) or (11). As we have no a priori knowledge on the exact form of relationship $h: X \rightarrow \kappa$, we use the statistical approach, i.e. the technique used for the SDB model, thus assuming $\kappa$ can be represented by model equations e.g. by Eq. (10) for linear model.

\subsection{Kinetics Multi-Scale (KMS) model}

The kinetics multi-scale (KMS) model is developed based on the Kinetics Hybrid model described by Eq. (3-7) and Eq. (12-13), but we use the general mass-energy balance over an element of reactor volume combined with a mixing model to replace the bioreactor model which is denoted by Eq. (1-2). The mixing model is implemented using Computational Fluid Dynamics (CFD) based on the $k-\varepsilon$ turbulence model (Dubey et al., 2006). This approach was 
used to describe the mixing mechanism in a bioreactor with sufficient accuracy (Ranade, 2002).

The $k$ - $\varepsilon$ turbulence model is normally used in order to describe the mixing behaviour and to compute the turbulence in the bioreactor. The energy dissipation is expressed as:

$$
\varepsilon=((\Delta P F u)) / m=(\Delta P u) /(x \rho)
$$

where $\Delta p$ denotes the pressure drop, $m$ the mass, $F$ the tube cross-section, $x$ the axial coordinate and $u$ the velocity vector field. Moreover, the fluid flow equations need to be solved for a constant density fluid (Bode, 1994). These consist of the continuity equation:

$$
\operatorname{div}(\rho u)=0
$$

and the transport equations:

$$
\begin{aligned}
& \operatorname{div}(\rho u k)=\operatorname{div}\left[\left(\mu_{e f f} / \sigma_{k}\right) \nabla k\right]+G-\rho \varepsilon \\
& \operatorname{div}(\rho u k)=\operatorname{div}\left[\left(\mu_{e f f} / \sigma_{\varepsilon}\right) \nabla \varepsilon\right]+\left(C_{1} G-C_{2} \rho \varepsilon\right)(\varepsilon / k)
\end{aligned}
$$

where $\nabla k$ and $\nabla \varepsilon$ denote the gradient of $k$ and $\varepsilon$, respectively. $k$ is the kinetic energy of turbulence at the point of interest.

On the other hand, the Eddy Viscosity model is used to solve complex turbulent flows. This model has proven to be a valuable tool in the predictions of turbulent flow-field (Gatski and Jongen, 2000). Therefore, it would be useful to utilize this model to predict the turbulent flow-field in the bioreactor.

The Eddy Viscosity is given by

$$
u_{T}=C_{\mu} \rho k^{2} / \varepsilon
$$

Note that $G$ is the scalar dissipation function $G=\tau_{i j} \tau_{i j} /\left(2 \mu_{e f f}\right)$ and the scalar values: $C_{\mu}=0.09, C_{1}=1.44, C_{2}=1.92, \sigma_{k}=1$ and $\sigma_{\varepsilon}=1.3$.

The Navier-Stokes equation is used for flow equations to describe the instantaneous behaviour of the turbulent liquid flow in ethanolic fermentation process. The resulting Reynolds equations and the continuity equation are given by: 
$\frac{\partial\left(\rho u_{i}\right)}{\partial t}+\frac{\partial\left(\rho u_{i} u_{j}\right)}{\partial x_{i}}=-\frac{\partial}{\partial x_{i}}\left(\tau_{i j}+\rho u_{i}^{\prime} u_{j}^{\prime}\right)-\frac{\partial p}{\partial x_{i}}+\rho g_{i}$

$\frac{\partial \rho}{\partial t}+\frac{\partial}{\partial x_{i}}\left(\rho u_{i}\right)=0$

For model accuracy and computational expense, a reasonable eddy viscosity models relating the individual Reynolds stresses to mean flow gradients are adopted:

$\rho u_{i}^{\prime} u_{j}^{\prime}=-\rho v_{t u r b}\left(\frac{\partial u_{i}}{\partial x_{j}}+\frac{\partial u_{j}}{\partial x_{i}}\right)+\frac{2}{3} \rho \partial_{i j} k$

where $v_{\text {turb }}$ is the turbulent eddy viscosity. The transport of momentum is thought of as turbulent eddies, which like molecules, collide and exchange momentum.

The general balance over an element of reactor volume is given by:

$\delta(\rho \phi) / \delta t+\delta\left(\rho U_{i} \phi\right) / \delta x_{i}=\delta\left(\Gamma_{\phi}(\delta \phi) / \delta x_{i}\right) / \delta x_{i}+S_{\phi}$

where $\rho$ is the density of fluid, $\phi$ is the concentration of any component, $U_{i}$ is the local velocity in the $x_{i}$-direction, $\Gamma_{\phi}$ is the effective diffusivity of $\phi$ and $S_{\phi}$ is the volumetric source term (rate of production of $\phi$ per unit volume). Note that, the reaction rates described by Eq. (13) are embedded into the source term $S_{\phi}$. Also note that the notation $\phi \equiv Z$ denotes the output variables i.e. optical density, substrate and ethanol concentrations.

Using the KMS model, we can compute the mass of substrate $\left(M S_{t_{b}}\right)$ and mass of product $\left(M S_{t_{b}}\right)$ at the end of batch time over the total reactor volume, i.e.:

$$
\begin{aligned}
& M S_{t_{b}}=\sum_{i=1}^{h}\left(S_{i} \Delta V_{i}\right) \\
& M P_{t_{b}}=\sum_{i=1}^{h}\left(P_{i} \Delta V_{i}\right)
\end{aligned}
$$

where $S_{i}$ is the substrate concentration at $i$ location, $V_{i}$ is the volume of mesh at $i$ location and the volume of medium in the reactor $V_{R}$ is given by

$V_{R}=\sum_{i=1}^{h} \Delta V_{i}$ 
Here $h$ is the number of discretizations (i.e. finite volume). Then, we can calculate as follows

$$
\Phi_{\delta}=\left[\begin{array}{c}
Y \\
P_{r}
\end{array}\right]=\left[\begin{array}{c}
100\left(M P_{t_{b}}-M P_{0}\right) /\left(M S_{0}-M S_{t_{b}}\right) \\
\left(M P_{t_{b}}-M P_{0}\right) / t_{b}
\end{array}\right]
$$

Here $M S_{0}$ and $M P_{0}$ correspond to the initial mass of substrate and mass of product (ethanol) in the fermentation medium, respectively.

\subsection{Model analysis and validation}

In this section, we develop the models using the proposed approaches, then the models are analysed using the ANOVA and validated against the experimental data.

The SDB model was developed using the input and output data as shown in Table 2, and the regression method results in the following quadratic model (Eq. 29) where the ANOVA analysis is presented in Tables 3 and 4:

$\left[\begin{array}{c}Y \\ P_{r}\end{array}\right]=\left[\begin{array}{c}33.098 \\ 0.234\end{array}\right]+\left[\begin{array}{cc}-18.785 & -0.143 \\ -0.139 & -9.9 \times 10^{-4}\end{array}\right]\left[\begin{array}{c}A R \\ S S\end{array}\right]+\left[\begin{array}{ll}A R & S S\end{array}\right]\left[\begin{array}{cc}0 & 0.147 \\ 1.02 \times 10^{-3} & 0\end{array}\right]\left[\begin{array}{c}A R \\ S S\end{array}\right]$

As shown in Tables 3 and 4, the ANOVA results of the model demonstrate that the model is highly significant, as indicated by the Fisher's $F$ test $\left(F_{\text {model }}=9.73\right.$ and 9.3$)$ and a low "Prob $>$ F" value $\left(P_{\text {model }}>0.0047\right.$ and $\left.>0.0054\right)$. Additionally, the goodness of the fit of the model is also checked by the determination coefficient $\left(R^{2}\right)$. In this case, the values of the determination coefficient $\left(R^{2}=0.8743\right.$ and $\left.R^{2}=0.8691\right)$ indicate that $87 \%$ of the sample variation in yield and productivity are well explained by the model. Thus, the SDB model is statistically adequate to predict the yield and productivity within the range of experimental setting.

For the kinetics hybrid model, solving the optimisation problem of $P_{2}$ gives the predicted Herbert's kinetic parameters as shown in Table 5. This set of data is then used to obtain the following regressed linear model by solving the optimisation problem of $P_{3}$ : 
$\kappa=\left[\begin{array}{l}k_{1} \\ k_{2} \\ k_{3} \\ k_{4} \\ k_{5} \\ k_{6}\end{array}\right]=\left[\begin{array}{c}1.4085-0.2852 X 1+0.3692 X 2 \\ 0.0010 \\ 0.6631-0.0148 X 1+0.0220 X 2 \\ 0.1040+0.0142 X 1+0.0128 X 2 \\ 0.7558-0.1019 X 1-0.0211 X 2 \\ 0.0143-0.0001 X 1-0.0019 X 2\end{array}\right]$

By combining the linear model of Eq. (30) with the macro scale bioreactor of Eq. (1-7), the kinetics hybrid (KH) model can be obtained. This $\mathrm{KH}$ model is then validated against another set of experimental data of AR and SS, which were chosen within the experimental ranges of AR and SS, i.e. 1.2 LPM AR and 175rpm SS. Figs. 1 to 3 show the results of model validation - prediction using the $\mathrm{KH}$ model as compared to experimental data. As observed, the kinetics hybrid model can fit the experimental data well.

Similarly, a series of simulations were performed by using Runge Kutta method to validate the KMS model, where a set of AR and SS was generated using the CCD technique. The ANOVA results of the fitness of the KMS model are shown in Tables 6 and 7.

The determination coefficients of the KMS model are $R^{2}=0.8353$ and $R^{2}=0.8021$, respectively for the yield and productivity. This result shows that more than $80 \%$ of the sample variation in yield and productivity are well explained by the model. Thus, statistically the model is sufficiently accurate in term of the prediction for yield and productivity within the experimental range.

\subsection{Optimisation of bioreactor's performances}

\subsubsection{Problem Formulation}

Our objective is to find the optimum AR and SS by maximizing the yield and productivity using the developed models, i.e. SDB, KH and KMS models. The optimisation problem is formulated as follows:

$P_{4}: \max _{X_{\min \leq x \leq x_{\max }}}\left\{\left(\phi^{T} \phi\right)\right\}$

Subject to: the model (either SDB, KH or KMS Model). 
To solve this optimisation problem, we can use a nonlinear programming technique, but in this study, we employ the RSM technique to find the optimum AR and SS.

\subsubsection{Optimisation Results}

After applying the RSM technique, a number of 3D surface plots were generated for finding the optimum AR and SS using the SDB model as shown in Fig. 4. From Fig. 4, we observe that there is a significant (quadratic) effect of AR and SS on the response surface. Within the experimental range, the optimum values of AR and SS maximizing yield and productivity are 1.47LPM and 242rpm, respectively. The maximum values of yield and productivity are 24.5\% and $\mathbf{0 . 2 g} / \mathbf{L . h r}$, respectively. This value of yield corresponds to $97.8 \%$ of the maximum theoretical value for yield of ethanol. In general, the response of yield increases as the SS increases from $150 \mathrm{rpm}$ to its peak value at $242 \mathrm{rpm}$. Additionally, the yield shows a significant increase with the increase in AR. Overall, the SDB model demonstrates a reasonable prediction of the impacts of $\mathrm{AR}$ and $\mathrm{SS}$ on the values of yield and productivity since the ANOVA results for SDB model, i.e. Tables 3 and 4 shows the significance of this model on yield and productivity.

For the KH model, the effects of AR and SS on the yield and productivity are shown in Fig. 5. The surface responses show that both yield and productivity are significantly affected by AR and SS. Also, it can be seen that the yield and productivity increase with the increase of AR and SS. Thus, this suggests that the KH model was able to capture the effect of both AR and SS on the yield and productivity. Just like the SDB model, the $\mathrm{KH}$ model is able to predict the impacts of AR and SS on the yield and productivity reasonably well.

The optimum values of $\mathrm{AR}$ and SS were obtained using the KH model as 1.43LPM and 250rpm, respectively. These optimum values lead to the maximum yield of $\mathbf{2 1 . 1 5 0 \%}$ and

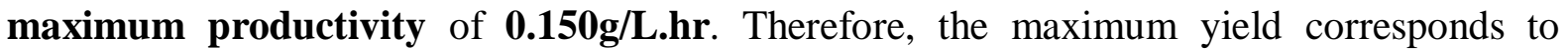
$96.6 \%$ of the maximum theoretical value for yield. Also, we note that the predicted maximum yield and productivity by the KH model are comparable with that of the SDB model.

Fig. 6 shows the response surface plots for the KMS model. Like other models, the KMS model shows that the yield and productivity increase with the increase of AR and SS. Within the experimental range, the optimum values of AR and SS are 1.45LPM and 240rpm, respectively. These values correspond to the maximum yield of $\mathbf{2 4 . 1 2 8 \%}$ and maximum productivity of $\mathbf{0 . 2 0 7} \mathrm{g} / \mathrm{L} . \mathbf{h r}$. It is interesting to note, though, that the response surface plots 
generated based on the KMS model are almost similar to those generated by the SDB model. In contrast, the response surface plots of the KMS model are quite different from those of the $\mathrm{KH}$ model. This is due to the assumption of well-mixing behaviour in the macro bioreactor model used in the original $\mathrm{KH}$ model.

\subsubsection{Experimental Validation}

In this section, we present the experimental validation of the optimum values of AR and SS found using different models. Tables 8 and 9 show the comparison of errors in the predictions of maximum yield and productivity using different models. The errors were calculated as:

$$
\begin{aligned}
& \text { ErrorYield }=100 \% \times \mid \text { Yield }_{\max }-\text { Yield }_{i} \mid / \text { Yield }_{\max } \\
& \text { Error } \operatorname{Pr} o d=100 \% \times\left|\operatorname{Pr} o d_{\text {max }}-\operatorname{Pr} o d_{i}\right| / \operatorname{Pr} o d_{\text {max }}
\end{aligned}
$$

Where the subscript $i$ refers to the $i$-model, i.e. $i \in\{S D B, K H, K M S\}$.

Note that the KMS model exhibits the best prediction of maximum experimental yield and productivity (lowest prediction error). Despite its simplicity, the SDB model predictions are relatively good and better than the $\mathrm{KH}$ model predictions. This means that by including the CFD model in the macro bioreactor model, the effect of mixing arising from the AR and SS can reasonably be captured by the KMS model - provide the most accurate prediction of yield and productivity. Meanwhile, the $\mathrm{KH}$ model is only capable of taking into account the effect of AR and SS within the context of well-mixing condition inside the bioreactor. The fact that $\mathrm{KH}$ model resulted in the largest error in the predictions of maximum experimental yield and productivity suggested that there was a significant deviation from ideal mixing inside the bioreactor. Interestingly, despite this significant deviation from the ideal mixing condition, the SDB model, which directly expressed the yield and productivity as a quadratic function of AR and SS, was shown to be capable of predicting the maximum yield and productivity with a sufficient accuracy i.e. less than $5 \%$ error.

To demonstrate that the non-ideal mixing conditions are occurring in the bioreactor, Fig. 7 shows an example of the mixing phenomena obtained from the CFD simulation under the operation of AR 1.47LPM and SS 242rpm. It was observed that both AR and SS have 
significant impacts towards the turbulence and mixing mechanism in the bioreactor. Thus, this simulation is showing the occurrence of non-ideal mixing behaviour, and explains the ability of the bioreactor models to capture the non-ideally mixing behaviour of the bioreactor, which is essential for a better prediction of yield and productivity. Further research will be done in order to fully evaluate this approach. Current study only shows the significant impacts of both AR and SS in terms of the bioreactor models developed in order to capture the non-ideally mixed behaviour of the bioreactor.

\section{Conclusions}

In this paper, we have proposed a new approach to the bioreactor modelling based on the expanded Herbert's kinetics concept. The developed models were able to incorporate the mixing mechanism by adopting two input variables, namely the aeration rate and stirrer speed. It was shown that the models could be used to optimize the bioreactor's performances. Furthermore, it was found from this study that the incorporation of mixing CFD model into the KH model of microbial kinetics (i.e. KMS modelling approach) could predict reasonably well (and optimize) the yield and productivity by adjusting the aeration rate and stirrer speed. It is important to note that using the conventional models of microbial kinetics it is not possible to optimize the yield and productivity using the AR and SS because of well-mixed assumption. As the results, the developed models could be used for studying the effects of $\mathrm{AR}$ and SS on the rates of growth, substrate consumption and product formation so that the $\mathrm{AR}$ and SS could be used as additional parameters to optimize the bioreactor.

\section{Acknowledgements}

The work described in this paper was supported by the eScienceFund project (No: 02-02-07SF0001) under the Ministry of Science, Technology and Innovation (MOSTI).

\section{References}

Bode, J., 1994. Computational fluid dynamics applications in the chemical industry. Computers chem. Engng 18, S247-S251.

Bezzo, F., Macchietto, S., Pantelides, C.C., 2003. General hybrid multizonal/CFD approach for bioreactor modeling. AIChE Journal 49, 2133-2148. 
Dubey, H., Das, S.K., Panda, T., 2006. Numerical simulation of a fully baffled biological reactor: The differential circumferential averaging mixing plane approach. Biotechnology and Bioengineering 95, $754-766$.

Gatski, T.B., Jongen, T., 2000. Nonlinear eddy viscosity and algebraic stress models for solving complex turbulent flows. Progress in Aerospace Sciences 36, 655-682.

Harvey, A.D., Rogers, S.E., 1996. Steady and unsteady computation of impeller-stirred reactors. AIChE Journal 42, 2701-2712.

Hjersted, J.L., Henson, M.A., 2006. Optimization of fed-batch Saccharomyces cerevisiae fermentation using dynamic flux balance models. Biotechnol. Prog. 22, 1239-1248.

Hutmacher, D.W., Singh, H., 2008. Computational fluid dynamics for improved bioreactor design and 3D culture. Trends in Biotechnology 26, 166-172.

Jenne, M., Reuss, M., 1999. A critical assessment on the use of k-e turbulence model for simulation of turbulent flow induced by a Ruston turbine in baffled stirred tank reactor. Chem. Eng. Sci. 54, 3921-3941.

Jiang, Y., Khadilkar, M.R., Al-Dahhan, M.H., Dudukovic, M.P., 2002. CFD of multiphase flow in packed-bed reactors: II. results and applications. AIChE Journal 48, 716-730.

Konde, K.S., Modak, J.M., 2007. Optimization of bioreactor using metabolic control analysis approach. Biotechnol. Prog. 23, 370-380.

Liew, E.W.T., Nandong, J., Samyudia, Y., 2009. Experimental investigation on the impact of aeration rate and stirrer speed on micro-aerobic batch fermentation. Journal of Applied Sciences 9, 3126-3130.

Lübbert, A., 1992. Advanced methods for bioreactor characterization. Journal of Biotechnology 25, 145-182.

Noordin, M.Y., Venkatesh, V.C., Sharif, S., Elting, S., Abdullah, A., 2004. Application of response surface methodology in describing the performance of coated carbide tools when turning AISI 1045 steel. Journal of Materials Processing Technology 145, 46-78.

Rahimi, M., Parvareh, A., 2005. Experimental and CFD investigation on mixing by a jet in a semi-industrial stirred tank. Chemical Engineering Journal 115, 85-92.

Ranade, V. V., 1997. An efficient computational model for simulating flow in stirred vessels: a case of Rushton turbine. Chemical Engineering Science 52, 4473-4484. 
Ranade, V.V., 2002. Computational flow modeling for chemical reactor engineering. Process Systems Engineering, UK.

Stanbury, P.F., Whitaker, A., Hall, S.J., 1995. Principles of fermentation technology. Butterworth-Heinemann, UK.

Starzak, M., Krzystek, L., Nowicki, L., Michalski, H., 1994. Macroapproach kinetics of ethanol fermentation by Saccharomyces cerevisiae: experimental studies and mathematical modelling. The Chemical Engineering Journal 54, 221-240.

Venneker, B.C.H., Derksen, J. J., Van den Akker, H.E.A., 2002. Population balance modeling of aerated stirred vessels based on CFD. AIChE Journal 48, 673-685.

Wadsworth, H.M., 1998. Handbook of Statistical Methods for Engineers and Scientists, second ed. McGrawHill.

Wiechert, W., 2002. Modeling and simulation: tools for metabolic engineering. Journal of Biotechnology 94 , $37-63$. 
Table 1

Input variables and their levels employed in two-factor factorial design

\begin{tabular}{lllllll}
\hline Factor & Variable & Units & $\begin{array}{l}\text { Low } \\
\text { Level (-) }\end{array}$ & $\begin{array}{l}\text { Middle } \\
\text { Level (0) }\end{array}$ & $\begin{array}{l}\text { High } \\
\text { Level (+) }\end{array}$ \\
\hline$X_{1}$ & $\begin{array}{l}\text { Aeration } \\
(\text { AR) }\end{array}$ & Rate & LPM & 1.0 & 1.25 & 1.5 \\
$X_{2}$ & $\begin{array}{l}\text { Stirrer } \\
(\text { SS) }\end{array}$ & Speed & rpm & 150 & 200 & 250 \\
\hline
\end{tabular}




\section{Table 2}

CCD matrix for the two independent variables (Aeration Rate and Stirrer Speed)

\begin{tabular}{ccccccc}
\hline $\begin{array}{c}\text { Standard } \\
\text { Order }\end{array}$ & Run Order & Block & $\begin{array}{c}\boldsymbol{X}_{\mathbf{1}}: \text { Aeration } \\
\text { Rate (LPM) }\end{array}$ & $\begin{array}{c}\boldsymbol{X}_{2}: \\
\text { Stirrer } \\
\text { Speed (rpm) }\end{array}$ & $\begin{array}{c}\boldsymbol{Y}_{1}: \\
\text { Yield (\%) }\end{array}$ & $\begin{array}{c}\boldsymbol{Y}_{2}: \\
\text { Productivity } \\
\text { (g/L.hr) }\end{array}$ \\
\hline 7 & 1 & 1 & 1.25 & 200 & 21.500 & 0.180 \\
1 & 2 & 1 & 1.0 & 150 & 14.788 & 0.099 \\
5 & 3 & 1 & 1.25 & 200 & 21.050 & 0.176 \\
6 & 4 & 1 & 1.25 & 200 & 21.250 & 0.178 \\
3 & 5 & 1 & 1.0 & 250 & 15.105 & 0.102 \\
4 & 6 & 1 & 1.5 & 250 & 24.040 & 0.160 \\
2 & 7 & 1 & 1.5 & 150 & 16.392 & 0.106 \\
13 & 8 & 2 & 1.25 & 200 & 24.000 & 0.230 \\
12 & 9 & 2 & 1.25 & 200 & 23.500 & 0.200 \\
14 & 10 & 2 & 1.25 & 200 & 22.000 & 0.190 \\
10 & 11 & 2 & 1.25 & 129.29 & 18.511 & 0.115 \\
9 & 12 & 2 & 1.60 & 200 & 22.250 & 0.195 \\
11 & 13 & 2 & 1.25 & 270.71 & 23.500 & 0.210 \\
8 & 14 & 2 & 0.90 & 200 & 20.500 & 0.165 \\
\hline
\end{tabular}


Table 3

ANOVA results on yield for SDB model

\begin{tabular}{lll}
\hline Source & $\begin{array}{l}\text { p-value } \\
\text { Prob }>\text { F }\end{array}$ & \\
& 0.0047 & Significant \\
$A-A R$ & 0.0117 & \\
$B-S S$ & 0.0059 & \\
$\mathrm{AB}$ & 0.0309 & \\
$\mathrm{~A}^{2}$ & 0.0341 & \\
$\mathrm{~B}^{2}$ & 0.0201 & \\
Residual & & \\
Lack of Fit & 0.0563 & Not Significant \\
\hline
\end{tabular}




\section{Table 4}

ANOVA results on productivity for SDB model

\begin{tabular}{lll}
\hline Source & $\begin{array}{l}\text { p-value } \\
\text { Prob }>\text { F }\end{array}$ & \\
\hline Model & 0.0054 & Significant \\
$A-A R$ & 0.0718 & \\
$B-S S$ & 0.0069 & \\
$\mathrm{AB}$ & 0.1978 & \\
$\mathrm{~A}^{2}$ & 0.0203 & \\
$\mathrm{~B}^{2}$ & 0.0035 & \\
Residual & & Not Significant \\
Lack of Fit & 0.2461 & \\
\hline
\end{tabular}


Table 5

Predicted Herbert's Kinetics Parameters

\begin{tabular}{|c|c|c|c|c|c|c|c|c|}
\hline Run Order & $\begin{array}{l}X_{I}: \\
A R \\
(\mathrm{LPM})\end{array}$ & $\begin{array}{l}X_{2}: \\
S S \\
(\mathrm{rpm})\end{array}$ & $\hat{k}_{1}$ & $\hat{k}_{2}$ & $\hat{k}_{3}$ & $\hat{k}_{4}$ & $\hat{k}_{5}$ & $\hat{k}_{6}$ \\
\hline 1 & 1.25 & 200 & 1.4085 & 0.0010 & 0.6631 & 0.1040 & 0.7558 & 0.0143 \\
\hline 2 & 1.0 & 150 & 1.3245 & 0.0010 & 0.6559 & 0.0770 & 0.8788 & 0.0163 \\
\hline 3 & 1.25 & 200 & 1.1257 & 0.0010 & 0.6533 & 0.0909 & 0.7252 & 0.0173 \\
\hline 4 & 1.25 & 200 & 1.2591 & 0.0010 & 0.6731 & 0.0879 & 0.7127 & 0.0179 \\
\hline 5 & 1.0 & 250 & 2.0629 & 0.0010 & 0.6999 & 0.1026 & 0.8366 & 0.0125 \\
\hline 6 & 1.5 & 250 & 1.4925 & 0.0010 & 0.6703 & 0.1310 & 0.6328 & 0.0123 \\
\hline
\end{tabular}


Table 6

ANOVA results on yield for the KMS model

\begin{tabular}{lll}
\hline Source & $\begin{array}{l}\text { p-value } \\
\text { Prob }>\text { F }\end{array}$ & \\
\hline $\begin{array}{l}\text { Model } \\
\text { Residual }\end{array}$ & 0.0115 & Significant \\
$\begin{array}{l}\text { Lack of Fit } \\
\text { Pure Error }\end{array}$ & 0.2003 & Not Significant \\
\hline
\end{tabular}


Table 7

ANOVA Results on productivity for the KMS model

\begin{tabular}{lll}
\hline Source & $\begin{array}{l}\text { p-value } \\
\text { Prob }>\mathbf{F}\end{array}$ & \\
\hline Model & 0.0328 & Significant \\
Residual & & \\
Lack of Fit & 0.1693 & Not Significant \\
Pure Error & & \\
\hline
\end{tabular}




\section{Table 8}

Comparisons of model predictions and experimental results for yield

\begin{tabular}{|c|c|c|c|c|c|}
\hline$i$-Model & $\begin{array}{l}\text { Optimum } \\
\text { Aeration Rate } \\
\text { (LPM) }\end{array}$ & $\begin{array}{l}\text { Optimum } \\
\text { Stirrer Speed } \\
\text { (rpm) }\end{array}$ & $\begin{array}{l}\text { Model } \\
\text { Predicted } \\
\text { Maximum } \\
\text { Yield (\%) }\end{array}$ & $\begin{array}{l}\text { Experimentally } \\
\text { Verified } \\
\text { Maximum Yield } \\
(\%) \text { Yield }_{i}\end{array}$ & $\begin{array}{l}\text { Error Yield } \\
(\%)\end{array}$ \\
\hline SDB & 1.47 & 242 & 24.495 & 23.720 & 3.46 \\
\hline KH & 1.43 & 250 & 21.150 & 20.950 & 14.73 \\
\hline KMS & 1.45 & 240 & 24.128 & $24.570^{\ell}$ & 1.80 \\
\hline
\end{tabular}




\section{Table 9}

Comparisons of model predictions and experimental results for productivity

\begin{tabular}{|c|c|c|c|c|c|}
\hline & & & & Experimentally & \\
\hline$i$-Model & $\begin{array}{l}\text { Optimum } \\
\text { Aeration Rate } \\
\text { (LPM) }\end{array}$ & $\begin{array}{l}\text { Optimum } \\
\text { Stirrer Speed } \\
\text { (rpm) }\end{array}$ & $\begin{array}{l}\text { Maximum } \\
\text { Productivity } \\
\text { (g/L.hr) }\end{array}$ & $\begin{array}{l}\text { Verified } \\
\text { Maximum } \\
\text { Productivity } \\
\text { (g/L.hr) } \text { Prod }_{i}\end{array}$ & $\begin{array}{l}\text { Error Prod } \\
(\%)\end{array}$ \\
\hline SDB & 1.47 & 242 & 0.198 & 0.185 & 11.90 \\
\hline KH & 1.43 & 250 & 0.150 & 0.148 & 29.52 \\
\hline KMS & 1.45 & 240 & 0.207 & $0.210^{\ell}$ & 1.43 \\
\hline
\end{tabular}




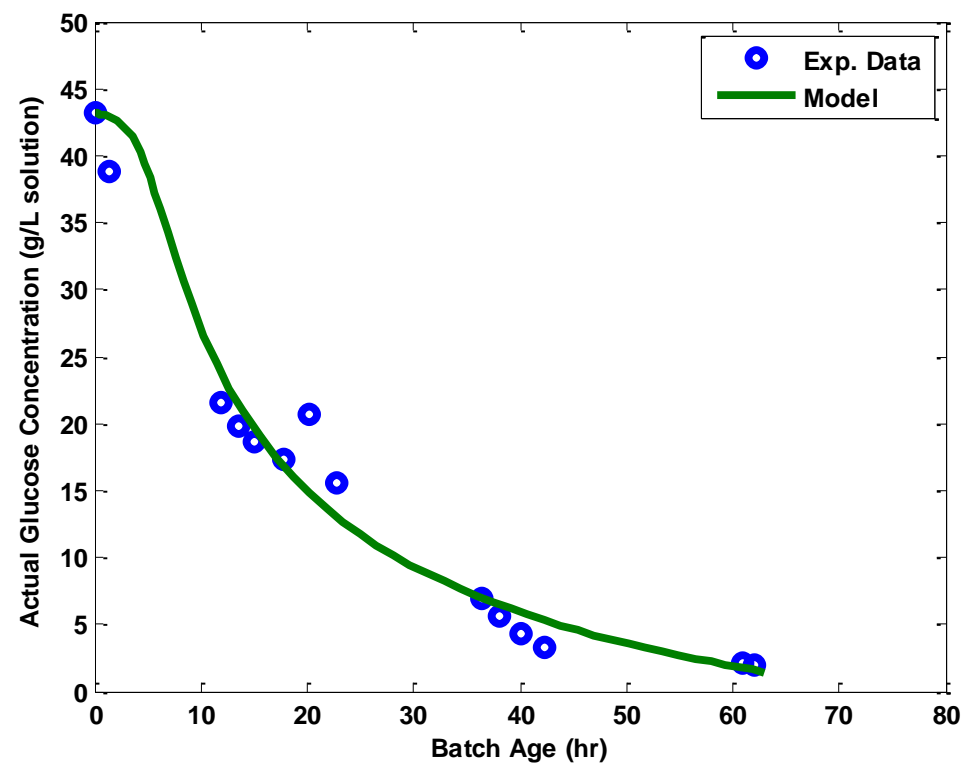

Fig. 1. Kinetics hybrid (KH) model fitting for actual glucose concentration (g/L solution) vs batch age (hr) for 1.2LPM AR and 175rpm SS 


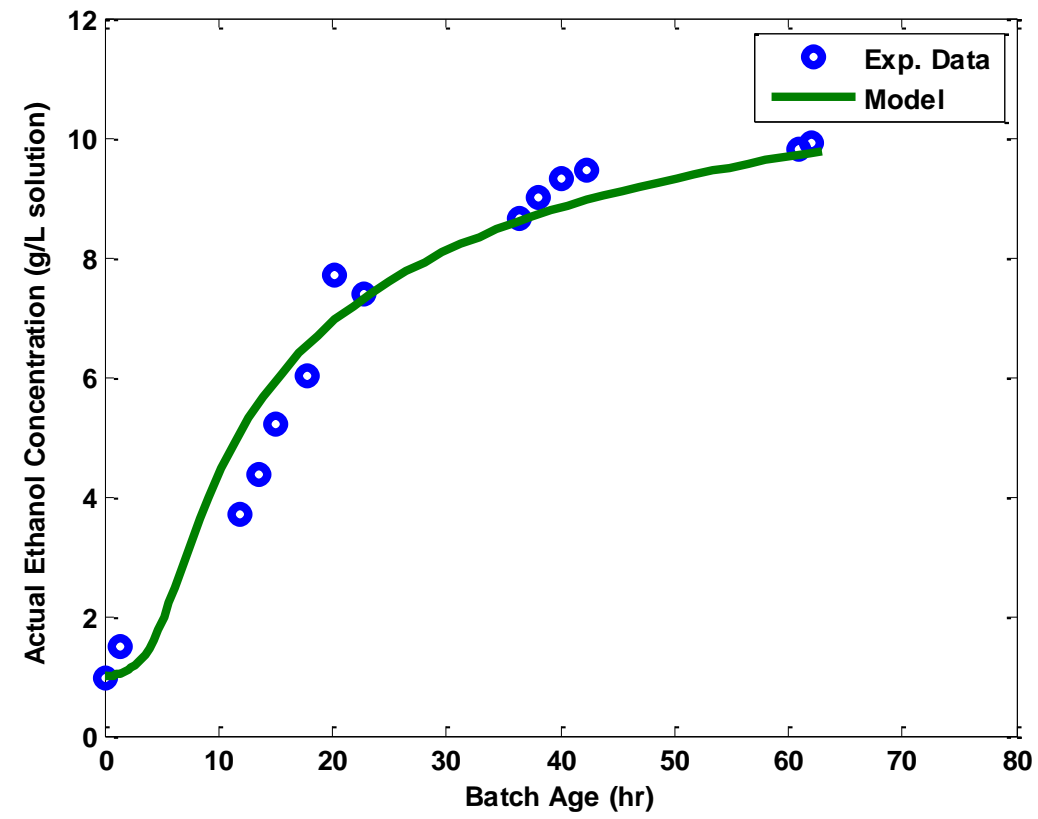

Fig. 2. Kinetic hybrid (KH) model fitting for actual ethanol concentration (g/L solution) vs batch age (hr) for 1.2LPM AR and 175rpm SS 


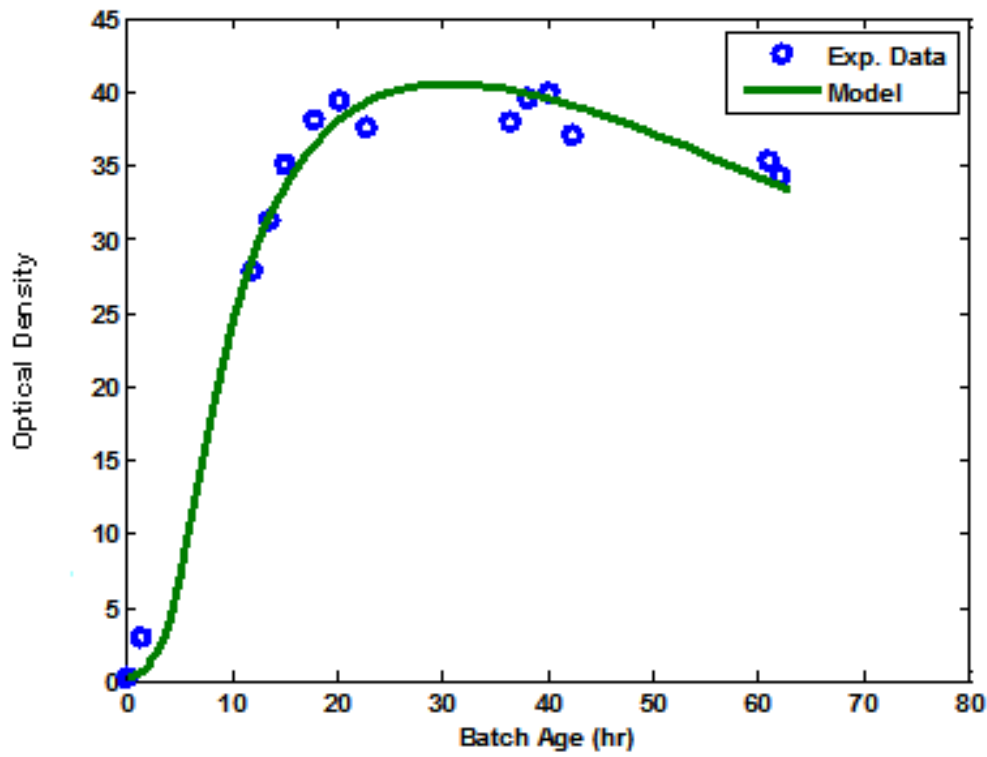

Fig. 3. Kinetics hybrid (KH) model fitting for optical density vs batch age (hr) for 1.2LPM AR and 175rpm SS 

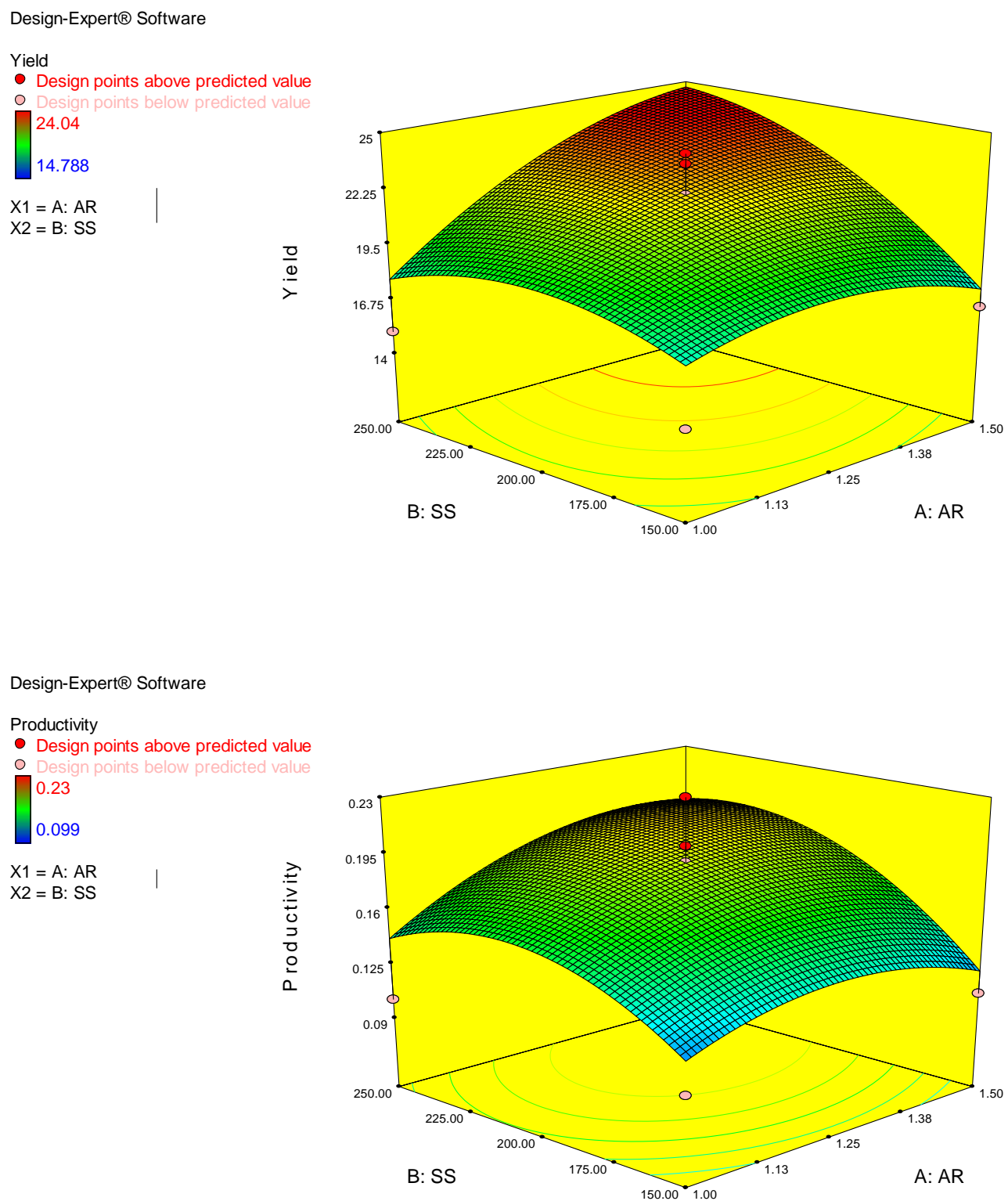

Fig. 4. Response surface plots for the SDB Model 
Design-Expert@ Software

Yield
21.099
12.006

$\mathrm{X} 1=\mathrm{A}: \mathrm{AR}$

$\mathrm{X} 2=\mathrm{B}: \mathrm{SS}$

Design-Expert@ Software

\section{Productivity \\ 0.144 \\ 0.083}

$\mathrm{X} 1=\mathrm{A}: \mathrm{AR}$

$\mathrm{X} 2=\mathrm{B}: \mathrm{SS}$
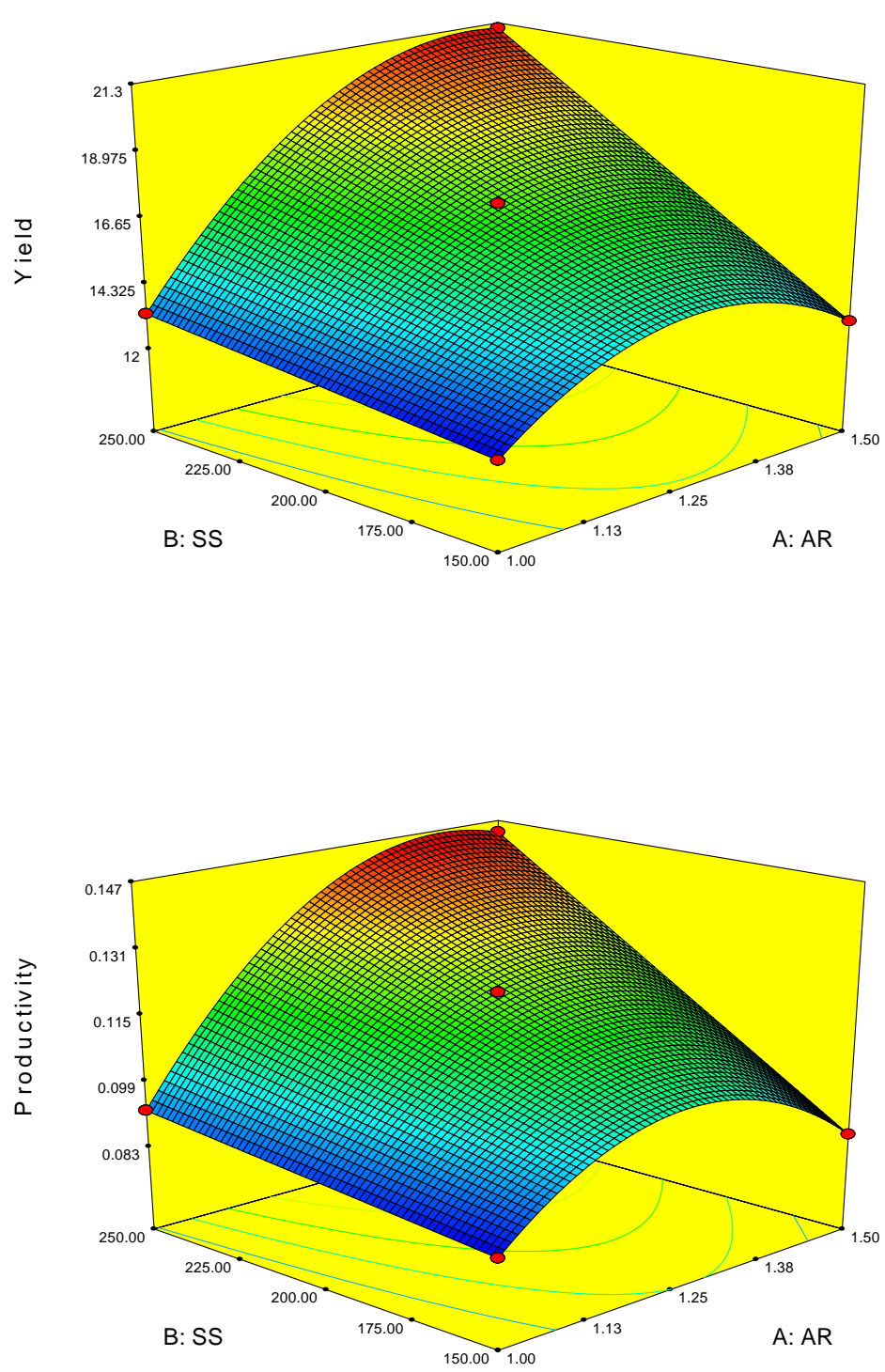

Fig. 5. Response surface plots for the KH Model 


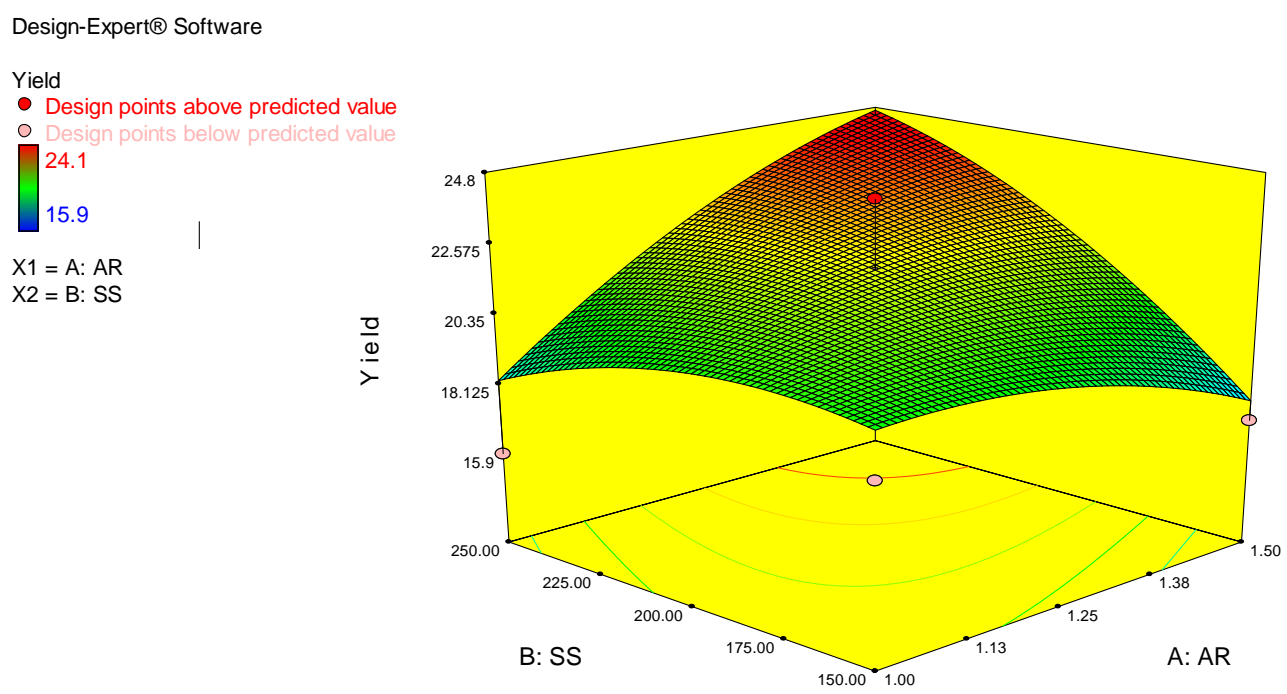

Design-Expert ${ }^{\circledR}$ Software

\section{Productivity}

- Design points above predicted value

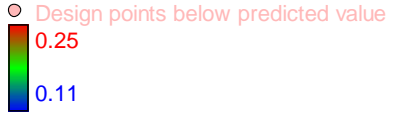

$\mathrm{X} 1=\mathrm{A}: \mathrm{AR}$

$\mathrm{X} 2=\mathrm{B}: \mathrm{SS}$

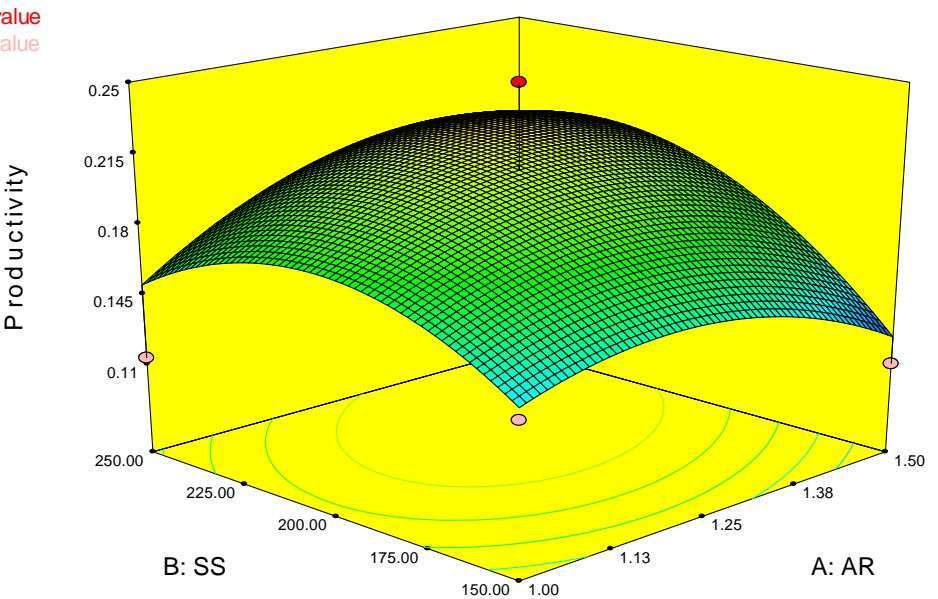

Fig. 6. Response surface plots for the KMS Model 

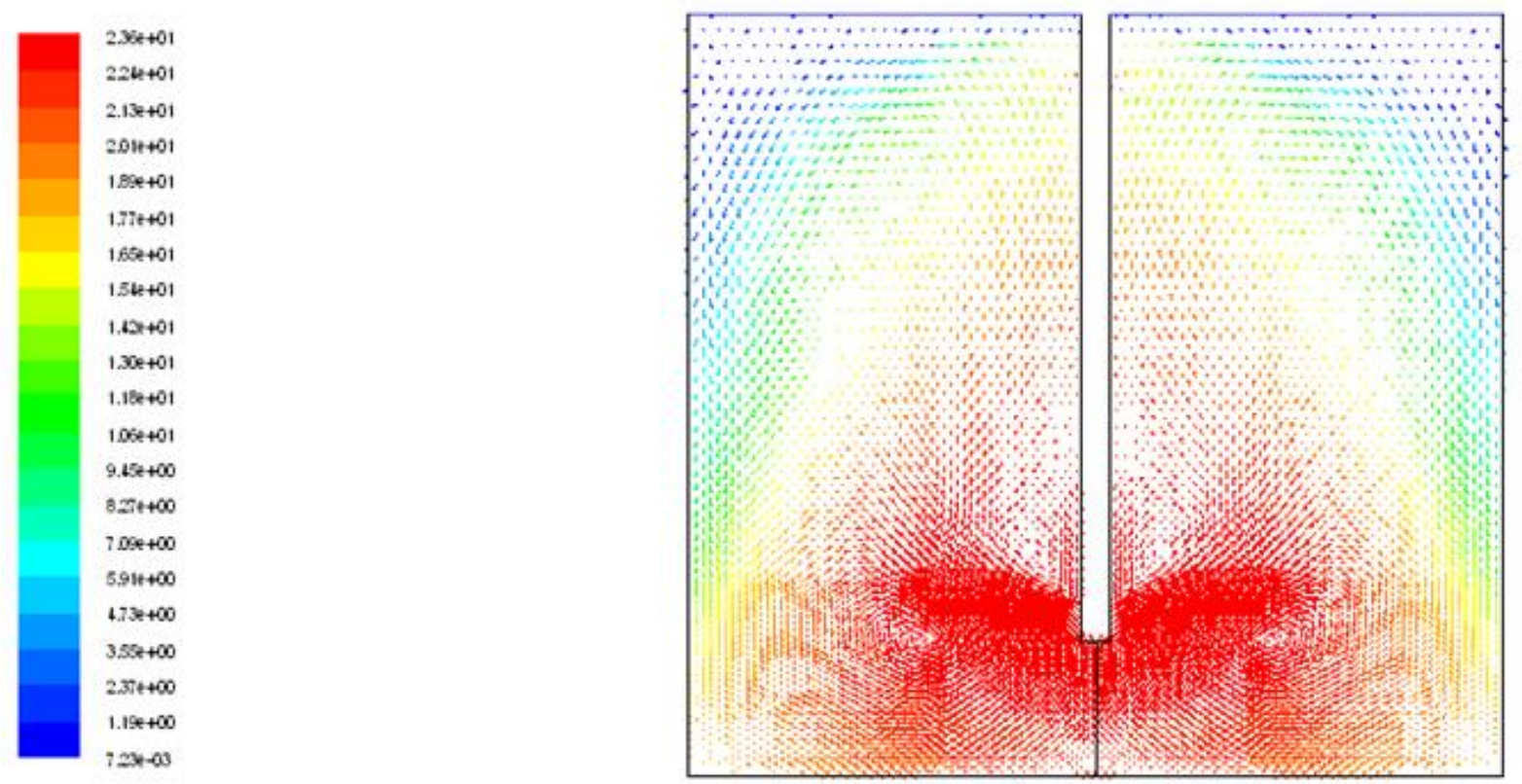

Fig. 7. CFD simulation of the mixing mechanism inside the bioreactor at AR of 1.47LPM and SS of 242rpm (Front view) 